CLINICAL STUDY

\title{
Operative treatment of prolactinomas: indications and results in a current consecutive series of 212 patients
}

\author{
J Kreutzer, R Buslei ${ }^{1}$, H Wallaschofski ${ }^{2}$, B Hofmann, C Nimsky, R Fahlbusch and M Buchfelder \\ Department of Neurosurgery, University of Erlangen-Nuremberg, Schwabachanlage 6, 91054 Erlangen, Germany, ${ }^{1}$ Institute of Neuropathology and \\ ${ }^{2}$ Division of Endocrinology and Metabolism, Department of Medicine, University of Erlangen-Nuremberg, Erlangen, Germany \\ (Correspondence should be addressed to J Kreutzer; Email: kreutzer@nch.imed.uni-erlangen.de)
}

\begin{abstract}
Objective: Medical therapy with dopamine agonists (DA) is the primary treatment of choice in most patients with prolactinomas. 'Classical' surgical indications are intolerance or lack of efficiency of DA therapy. Focusing on a possible shift of recent indications, we retrospectively analyzed our results of surgical treatment in prolactinomas.

Patients and methods: Between 1990 and 2005, we have operated on 212 consecutive patients with prolactinomas. Surgical indications were divided into 'classical' indications and 'modern' indications defined as cystic prolactinomas or patients with microprolactinomas who individually decided on a primary surgical treatment.

Results: Initial overall remission was accomplished in 53.2\% including giant prolactinomas. However, in microadenomas, the remission rate was significantly higher with $91.3 \%$. Overall remission at the latest follow-up was $42.7 \%$, but $72.5 \%$ in intrasellar tumors, $80 \%$ in cystic prolactinomas, and $84.8 \%$ in microprolactinomas. The overall recurrence rate was $18.7 \%$. Relapse of hyperprolactinemia in microprolactinomas was $7.1 \%$. In our series, continually less patients were surgically treated for 'classical' indications. By contrast, the number of patients who individually decided on a primary surgical therapy has increased considerably.

Conclusion: Remission rates after surgical treatment of prolactinomas remain excellent, particularly in microadenoma and intrasellar macroadenomas, whereas morbidity of transsphenoidal surgery is low in the hands of experienced pituitary surgeons. Our remission rates not only confirm the already interdisciplinarily accepted surgical indications, but also emphasize the value of primary transsphenoidal surgery as a discussion-worthy alternative to dopaminergic therapy in young patients with microprolactinomas or cystic tumors.
\end{abstract}

European Journal of Endocrinology 158 11-18

\section{Introduction}

Prolactin (PRL)-secreting pituitary adenomas represent the most frequent pituitary tumors with a predominance in premenopausal females aged $20-50$ years (1). Women were mostly diagnosed early because of a secondary amenorrhea and galactorrhea in $75 \%$ (2). At the time of diagnosis, they often exhibit microprolactinomas. Dopamine agonists (DA) have been recognized to be effective in the medical treatment of prolactinomas since the beginning of the 1970s (3, 4). Bromocriptine, an ergot derivative that binds to and stimulates dopamine (D2) receptors on lactotroph cells, represents the initial and therefore reference compound. It has proved to be effective in suppressing PRL secretion, reducing prolactinoma size and restoring gonadal function in many studies (2-7). In a collaborative European multicenter study on 459 women with prolactinomas, normoprolactinemia was achieved with bromocriptine in $59 \%$ (8). However, up to $12 \%$ of these patients did not tolerate the drug in therapeutic doses because of side effects and 5-10\% of patients showed only minimal or no response after bromocriptine therapy $(8,9)$. Cabergoline, a long-acting dopamine agonist, has been shown to be more effective (83\%) (8) than bromocriptine with a marked reduction in adverse effects $(7,8,10-15)$. Thus, cabergoline and also quinagolide started to progressively replace bromocriptine in the medical therapy of prolactinomas during the last decade (16-20). Nevertheless, the Kaplan-Meier estimates of the 5-year recurrence rates after withdrawal of cabergoline therapy in microprolactinomas and macroprolactinomas in a large prospective study by Colao et al. (21) were 26 and 33\% respectively. Moreover, the recurrence rate in the subgroup of patients with tumor remnants on magnetic resonance imaging (MRI) before withdrawal, increased to 42 and $78 \%$ in micro- and macroadenomas respectively. Biswas et al. retrospectively reported a relapse of hyperprolactinemia in $64 \%$ of patients after withdrawal 
of either cabergoline or bromocriptine (22). Tumor re-expansion after withdrawal is more uncommon and seen in about $20 \%$ of cases (23).

However, primary medical therapy with DA undoubtly is currently established as the 'gold-standard' treatment in patients with prolactinomas. In contrast, transsphenoidal surgery is currently only seen as a secondline treatment, although the published surgical rates of PRL level normalization in specialized centers, especially for microprolactinomas, are excellent and at least comparable with dopamine agonist therapy varying between 67 and $88 \%$ (24-34).

On the other hand, transsphenoidal surgery for prolactinomas is accepted for a distinct number of indications, namely non-responders to medical therapy, intolerance of DA, cerebrospinal fluid (CSF) fistulas due to tumor shrinkage after DA therapy and neurological deficits, such as rapid loss of vision or cranial nerve palsies due to tumor hemorrhage or apoplexy (35). All in all primary surgical treatment of microprolactinomas and tumors with dominant cystic parts unlikely to shrink under DA therapy remain controversial so far. This is certainly even more true for the subgroup of young patients who individually decide on a primary surgical treatment in a specialized pituitary surgery centre as an alternative an to a potential long-term medical therapy. Therefore, our purpose was to retrospectively analyze our consecutive surgical series since 1990 in order to confirm the efficacy and safety of transsphenoidal surgery for prolactinomas in the hands of dedicated pituitary surgeons. We focused on microadenomas and investigated changes in indications in order to reflect the impact of modern DA throughout the last 15 years and to potentially re-define clinical decision pathways in the interdisciplinary management of prolactinomas.

\section{Patients and methods}

We retrospectively analyzed our consecutive series of 212 patients who underwent surgery for PRL-secreting pituitary adenomas between August 1990 and August 2005. All cases of residual and/or recurrent adenomas previously operated on were excluded (complete series $n=232$ ). Within this period, we surgically treated a total of 1826 patients with pituitary adenomas by transsphenoidal surgery. Thus, prolactinomas constitute $12.7 \%$ of our operative series.

The consecutive series consisted of 133 female and 79 male patients. The mean age of the patients was 36 years (median age 32 years) with a range from 12 to 69 years. Fifty-six patients $(26.4 \%)$ harbored microadenomas $(<10 \mathrm{~mm}), 146$ patients $(68.9 \%)$ had macroadenomas $(>10 \mathrm{~mm})$, and 10 patients had giant adenomas $(>40 \mathrm{~mm})$. All patients underwent transsphenoidal surgery, except those with giant adenomas who received a combined transsphenoidaltranscranial management in two separate, consecutive operative procedures.
The diagnosis PRL-secreting pituitary adenomas was postoperatively confirmed in all tumors by immunohistochemical analysis (see below). Medical therapy with DA (mainly bromocriptine until end of the 1990s, thereafter increasingly cabergoline) had been started by the referring endocrinologists in 175 cases $(82.5 \%)$ but was aborted at least 4 weeks before surgery.

\section{Hormonal evaluation}

Fasting basal levels of PRL, cortisol, growth hormone (GH), testosterone, gonadotropins, estradiol, and thyroid hormones were measured in the serum of all patients preoperatively. Additionally, cortisol levels were measured 30 min after administration of adrenocorticotropin (ACTH). This endocrinological evaluation including morning fasting basal hormone levels and a short ACTH test was repeated at day 7 after transsphenoidal surgery and during follow-up visits at 3 months and then in annual or biannual intervals in 171 of $212(80.7 \%)$ patients with a mean follow-up of 19.6 months (range 3-132 months; median 12 months).

Additionally, 27 patients received testing of the hypothalamic-pituitary-adrenal axis by means of insulininduced hypoglycemia and insulin-like growth factor-I (IGF-I) preoperatively. Insulin-induced hypoglycemia was repeated postoperatively in four patients. Four different patients underwent stimulation by administration of GH-releasing hormone-arginine and another four patients received a combined releasing-hormone stimulation test of anterior pituitary function by administration of ACTH, luteinizing hormone-releasing hormone (LH-RH) and thyrotrophin-releasing hormone.

Initial remission was defined as a fasting morning basal PRL level $<500 \mu \mathrm{U} / \mathrm{ml}(23.6 \mathrm{ng} / \mathrm{ml})$ without dopaminergic therapy for at least 4 weeks before surgery on day 7 after surgery. Follow-up remission was defined as a morning fasting basal PRL level $<500 \mu \mathrm{U} / \mathrm{ml}$ without dopaminergic therapy for at least 3 months at the latest follow-up.

Male hypogonadism was defined by clinical manifestations, a testosterone level $<2.8 \mathrm{ng} / \mathrm{ml}$ and subnormal levels of gonadotropins. ACTH/cortisol deficiency was defined by clinical manifestations and a morning fasting basal cortisol level $<6 \mu \mathrm{g} / \mathrm{dl}$ with an inadequate increase after administration of ACTH $<15.0 \mu \mathrm{g} / \mathrm{dl}$. GH deficiency was determined by a GH level $<3.0 \mathrm{ng} / \mathrm{ml}$ after insulin-induced hypoglycemia and/or an age- and gender-adjusted low IGF-I level. Secondary hypothyroidism was defined by a free thyroxine $\left(\mathrm{T}_{4}\right)$ level $<10.0 \mathrm{mmol} / \mathrm{l}$ and a basal thyroid-stimulating hormone (TSH) level $<0.3 \mu \mathrm{U} / \mathrm{ml}$ in the absence of thyroid replacement therapy.

\section{Hormonal assays}

The assays used for hormone measurements changed over the long time period of the study. Between August 
1990 and December 2000, ELISAs were used (Pharmacia \& Upjohn). Since December 2000, a sequential chemiluminescent-immunometric assay was used (Immulite; DPC, Los Angeles, CA, USA). Assay normal values for PRL in healthy males or females are 17 or $25 \mathrm{ng} / \mathrm{ml}$ (97.5\% percentile) respectively. PRL conversion factor $\mathrm{ng} / \mathrm{ml} \times 21.2 \rightarrow \mu \mathrm{U} / \mathrm{ml}$. The inter-assay variation coefficient (CV) for PRL (serum \# 1-5) is 6.9, 8.2, 7.4, 5.9, 6.9, and $7.9 \%$.

\section{Histopathological evaluation}

Surgical specimens from all patients were analyzed according to the World Health Organisation guidelines using hematoxylin and eosin staining as well as immunohistochemistry. None of the pituitary adenomas used in this analysis were from patients diagnosed with multiple endocrine neoplasia type I.

Immunostaining of specimens was performed using the following panel of antibodies: ACTH (clone 02A3, DAKO), follicle-stimulating hormone (FSH; clone $\mathrm{C} 10$, DAKO), human GH (hGH; DAKO), LH (clone C93, DAKO), PRL (DAKO), and (TSH; clone 0042, DAKO).

Histopathological grading was based on the revised WHO classification system of tumors of endocrine organs (36).

\section{Subgroups of surgical indications}

Retrospective analysis of indications for the operative treatment of prolactinomas in our series revealed several patient subgroups. Indication subgroup 1 consisted of 38 patients with intolerable side effects due to dopaminergic therapy. Additionally, there were non-responders to DA therapy with regard to normalization of hyperprolactinemia (subgroup $2 ; n=27$ ) or inadequate tumor shrinkage (subgroup $3 ; n=52$ ). There were a further 22 patients presenting either with cranial nerve palsies due to involvement of the cavernous sinus, CSF fistulas after therapy with DA or signs of an acute space-occupying tumor hemorrhage on MRI with or without previous medical therapy. These patients were excluded (22 out of 212 patients; $10.4 \%$ of the series) from the surgical indications analysis, because they all represented surgical emergency cases independent from the time periods and the type of dopaminergic drug. Thus, we finally defined three 'classical' indication subgroups for operative treatment of prolactinomas.

By contrast the retrospective analysis revealed two subgroups of patients that we defined as 'modern' indications for transsphenoidal surgery for prolactinomas consisting of patients $(n=23)$ who individually decided on a primary surgical treatment (subgroup 4) and patients $(n=50)$ with cystic tumors $(80 \%$ of tumor volume according to the preoperative MRI T2-sequences) that are unlikely to shrink sufficiently under dopamine agonist therapy (subgroup 5).
Our surgical series of prolactinomas spans a time period of 15 years. In order to detect possible changes among the subgroups during this time period, we defined shorter periods of 5 years each, covering the beginning of the 1990s (time period A: 1990 to mid-1995), the second half of the 1990s (time period B: mid-1995 to mid-2000) and the last 5 years (time period C: mid-2000 to 2005). Within the time periods A to $\mathrm{C}$ (excluding 22 patients from indication subgroup 4 , see above), we treated 62,79 , and 49 patients respectively (Table 3 ).

There was no change in the specialized neurosurgical team (the two senior authors R Fahlbusch and $\mathrm{M}$ Buchfelder) performing the operative procedures during the series time period which possibly would have caused a bias with regard to the number and/or a preselection of patient subgroups by the referring endocrinologists.

\section{Statistical analysis}

Student's $t$-test for unpaired data was used to compare continuous variables among subgroups. Multiple logistic regression analysis was used to determine independent predictors of outcome/remission. Calculations were performed using the statistical software Statistica (StatSoft Inc., Tulsa, OK, USA).

\section{Results}

\section{Remission and recurrence}

Overall initial remission after selective adenomectomy with the dopaminergic therapy aborted at least 4 weeks before surgery was achieved in 102 of 212 patients (48.1\%) also including giant prolactinomas. Table 1 keeps records about the initial remission rates sorted by adenoma subgroups (intrasellar, is; intra- and suprasellar without visual compromise, $s 1$; intra- and suprasellar with visual deficits, s2; parasellar and/or sphenoidal, $p s / s p h$ and giant adenomas). The complete series included 61 (28.8\%) invasive tumors confirmed by the intraoperative findings concerning the cavernous sinus and/or sphenoid sinus.

Forty-one patients were lost to follow-up. Thus, data from our own laboratory utilizing the same assay were available for 171 patients. In these patients, overall initial remission was achieved in 91 patients (53.2\%) including giant prolactinomas. Initial remission was accomplished in 42 patients $(82.4 \%)$ sorted by growth extension, with intrasellar prolactinomas, 33 patients $(63.5 \%)$ with s1-adenomas, 6 patients $(18.8 \%)$ with s2-prolactinomas, 10 patients $(38.5 \%)$ with sphenoidal and/or parasellar extending prolactinomas, and in no patient harboring a giant prolactinoma (Table 1).

At the latest follow-up, the overall remission rate in the absence of dopaminergic therapy was $42.7 \%$ including giant adenomas. Again sorted by growth extension, follow-up remission was accomplished in $72.5 \%$ (is), 
Table 1 Initial remission rates (complete series $n=212$ ) and initial remission rates, follow-up remission rates and recurrence rates (follow-up series $n=171$ ) sorted by growth extension of prolactin (PRL)-secreting pituitary adenomas.

\begin{tabular}{|c|c|c|c|c|c|c|}
\hline Tumor extension $^{\mathrm{a}}$ & is & s1 & s2 & ps/sph & Giant & $\Sigma$ \\
\hline Number of tumor (complete series) & 64 & 64 & 37 & 37 & 10 & 212 \\
\hline Remission $^{\mathrm{b}}$ & $50 / 64$ & $38 / 64$ & $5 / 37$ & $9 / 37$ & $0 / 10$ & $102 / 212$ \\
\hline Initial remission rate ${ }^{b}$ & $78.1 \%$ & $59.4 \%$ & $13.5 \%$ & $24.3 \%$ & $0 \%$ & $48.1 \%$ \\
\hline Number of tumors (follow-up series) & 51 & 52 & 32 & 26 & 10 & 171 \\
\hline Initial remission ${ }^{\mathrm{b}}$ & $42 / 51$ & $33 / 52$ & $6 / 32$ & $10 / 26$ & $0 / 10$ & $91 / 171$ \\
\hline Initial remission rate ${ }^{b}$ & $82.4 \%$ & $63.5 \%$ & $18.8 \%$ & $38.5 \%$ & $0 \%$ & $53.2 \%$ \\
\hline Follow-up remission ${ }^{c}$ & $37 / 51$ & $24 / 52$ & $4 / 32$ & $8 / 26$ & $0 / 10$ & $73 / 171$ \\
\hline Follow-up remission rate ${ }^{c}$ & $72.5 \%$ & $46.1 \%$ & $12.5 \%$ & $30.8 \%$ & $0 \%$ & $42.7 \%$ \\
\hline Recurrence & $5 / 42$ & $8 / 33$ & $2 / 6$ & $2 / 10$ & - & $17 / 91$ \\
\hline Recurrence rate & $11.9 \%$ & $24.2 \%$ & $33.3 \%$ & $20.0 \%$ & - & $18.7 \%$ \\
\hline
\end{tabular}

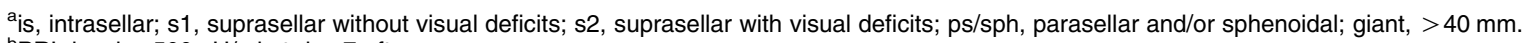

${ }^{\mathrm{b}} \mathrm{PRL}$ level $<500 \mu \mathrm{U} / \mathrm{ml}$ at day 7 after surgery.

${ }^{\mathrm{C} P R L}$ level $<500 \mu \mathrm{U} / \mathrm{ml}$ at latest follow-up in absence of dopaminergic therapy.

$46.1 \%(\mathrm{~s} 1), 12.5 \%(\mathrm{~s} 2), 30.8 \%$ (ps/sph), and $0 \%$ (giant) of tumors respectively (Table 1). Thus, a relapse of hyperprolactinemia occurred in 17 of 91 follow-up patients who were initially in remission (18.7\%). The recurrence rates sorted by growth extension adenoma subgroups were $11.9 \%$ (is), $24.2 \%$ (s1), 33.3\% (s2), and $20.0 \%$ (ps/sph) of tumors respectively (Table 1). In the subgroup of patients with cystic intrasellar and suprasellar (s1) adenomas, remission was more favorable with $80.0 \%$.

In the subcategory on microprolactinomas ( 56 of 212 tumors), initial remission was achieved in $83.9 \%$. The remission rate was even higher in purely intrasellarlocated microadenomas with $86.4 \%$ (Table 2).

Follow-up data from our own laboratory were eventually available in 46 patients with microprolactinomas. The initial remission rates for the subgroups of purely intrasellar microprolactinomas, the s1-microprolactinomas, and the microprolactinomas with sphenoidal and/or parasellar extension were $92.3,83.3$ and $100 \%$ respectively. Thus, overall initial remission in the follow-up series was $91.3 \%$ (Table 2). At the latest follow-up visit,

Table 2 Initial remission rates (complete series $n=56$ ) and initial remission rates, follow-up remission rates, and recurrence rates (follow-up series $n=46$ ) in microprolactinomas.

\begin{tabular}{|c|c|c|c|c|}
\hline Tumor extension $^{\mathrm{a}}$ & is & s1 & ps/sph & $\Sigma$ \\
\hline $\begin{array}{l}\text { Number of tumor } \\
\text { (complete series) }\end{array}$ & 44 & 10 & 2 & 56 \\
\hline Remission ${ }^{\mathrm{b}}$ & $38 / 44$ & $7 / 10$ & $2 / 2$ & $47 / 56$ \\
\hline Initial remission rate ${ }^{\mathrm{b}}$ & $86.4 \%$ & $83.3 \%$ & $100 \%$ & $83.9 \%$ \\
\hline $\begin{array}{l}\text { Number of tumors } \\
\text { (follow-up series) }\end{array}$ & 39 & 6 & 1 & 46 \\
\hline Initial remission ${ }^{\mathrm{b}^{\prime}}$ & $36 / 39$ & $5 / 6$ & $1 / 1$ & $42 / 46$ \\
\hline Initial remission rate ${ }^{\mathrm{b}}$ & $92.3 \%$ & $83.3 \%$ & $100 \%$ & $91.3 \%$ \\
\hline Follow-up remission ${ }^{c}$ & $34 / 39$ & $4 / 6$ & $1 / 1$ & $39 / 46$ \\
\hline Follow-up remission rate ${ }^{c}$ & $87.2 \%$ & $66.7 \%$ & $100 \%$ & $84.8 \%$ \\
\hline Recurrence & $2 / 36$ & $1 / 5$ & $0 / 1$ & $3 / 42$ \\
\hline Recurrence rate & $5.6 \%$ & $20.0 \%$ & $0 \%$ & $7.1 \%$ \\
\hline
\end{tabular}

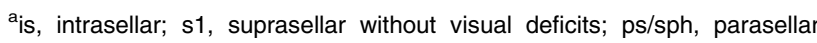
and/or sphenoidal.

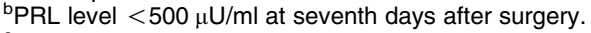

${ }^{\mathrm{c}} \mathrm{PRL}$ level $<500 \mu \mathrm{U} / \mathrm{ml}$ at latest follow-up in absence of dopaminergic therapy. remission in the absence of dopaminergic therapy was accomplished in $87.2 \%$ (is micro), $66.7 \%$ ( $s 1$ micro), and $100 \%$ ( $\mathrm{s} / \mathrm{sph}$ micro) resulting in an overall recurrence rate for microprolactinomas in $7.1 \%$. The most favorable recurrence rate was achieved in purely intrasellar microprolactinomas with $5.6 \%$ (Table 2).

There was a statistical significant difference between the mean preoperative PRL level in the remission group $9186 \pm 29132 \mu \mathrm{U} / \mathrm{ml}$ (median $2703 \mu \mathrm{U} / \mathrm{ml}$ ) and the preoperative PRL level in the persistent group (mean $100988 \pm 291454 \mu \mathrm{U} / \mathrm{ml}$; median $19650 \mu \mathrm{U} / \mathrm{ml}$ ). Thus, we also found a strong negative correlation between the height of preoperative PRL level and the chance of remission $(r=-0.21 ; P<0.002)$ by means of multiple logistic regression analysis. Tumor size (macro- versus micro-adenomas) was identified as an independent predictor of outcome/remission as well as adenoma growth extension subgroups (is, s1, s2, ps/sph, giant) with $r=-$ $0.44(P<0.001)$ and $r=-0.41(P<0.001)$ respectively.

\section{Morbidity, mortality, and pituitary deficiency}

As mentioned above, follow-up data was available in 171 patients (64 males and 107 females). Focusing on this group, preoperative hormonal evaluation demonstrated anterior pituitary deficiency of at least one axis in 52 of $64(81.2 \%)$ male patients. Besides hyperprolactinemia, we found secondary hypogonadism in $38(59.3 \%)$ patients (32 with no other anterior pituitary deficiency). Fifteen male patients (23.4\%) exhibited a secondary hypothyroidism. In 10 male patients, we found an ACTH/cortisol deficiency. There were two male patients with $\mathrm{GH}$ deficiency.

Out of 52 male patients, 14 (26.9\%) demonstrated an improvement of pituitary function on day 7 after transsphenoidal surgery and $18(34.6 \%)$ at the latest follow-up visit. On the other hand, of 64 patients, 4 (6.2\%) were diagnosed with new pituitary deficiencies at immediate post-operative evaluation and 11 patients (17.1\%) demonstrated new pituitary deficiencies at follow-up. All 11 patients with new pituitary deficiencies 
had macroadenomas ( $1: n=2 ; s 2: n=5 ; p s / s p h: n=2)$ or giant $(n=2)$ adenomas.

Next to the hyperprolactinemia and the clinical manifestations of hypogonadism (amenorrhea and/or galactorrhea), six female patients had secondary hypothyroidism, seven female patients demonstrated hypocortisolism and two female patients showed a GH deficiency. In these female patients, the pituitary functions improved in 5 of 13 cases $(38.5 \%)$ at the first postoperative hormonal evaluation at day 7 after transsphenoidal surgery and in 7 of 13 female patients (53.8\%) at the latest endocrinological follow-up. There was only one new onset of ACTH/cortisol deficiency out of 107 cases $(0.9 \%)$ in a female patient with an $s 2$-macroadenoma.

In conclusion, there was no mortality in this series. Morbidity excluding new anterior pituitary deficits was low with $3.8 \%$ (whole series) and very low in microprolactinomas with $1.8 \%$ ( 1 of 56 patients with microproloactinomas developed a febrile sinusitis). In detail, morbidity of the complete series consisted of one patient with a nasal bleeding requiring operative electrocauterization, two patients with postoperative meningitis, one patient with a deep venous thrombosis, two patients with febrile sinusitis, and two patients with a postoperative CSF leak. There was no new permanent diabetes insipidus. Postoperative transient hyponatremia with clinical symptoms (syndrome of inappropriate antidiuretic hormone secretion, SIADH) occurred in 10 patients $(4.7 \%)$.

At follow-up, new anterior pituitary deficiency had occurred in 12 of 171 patients $(7.0 \%$, all macro- or giant adenomas), while pituitary functions excluding hyperprolactinemia had improved in 25 of 171 patients (14.6\%).

\section{Analysis of surgical indications}

The indication subgroup 1 formed by patients with serious, intolerable side effects of dopaminergic therapy consisted of $38(20.0 \%)$ cases with a distribution among the time periods $\mathrm{A}$ to $\mathrm{C}$ of $24.2,20.3$, and $14.3 \%$ respectively (Fig. 1 and Table 3 ).
Subgroup 2 consisted of $27(14.2 \%)$ patients in whom DA therapy was not able to establish PRL levels within the normal ranges or a mild persistent hyperprolactinemia two- to threefold. Operative therapy had been necessary because of this 'classical' indication during time periods $\mathrm{A}$ to $\mathrm{C}$ in $21.0,12.7$, and $8.2 \%$ of cases respectively (Fig. 1 and Table 3 ).

Inadequate tumor shrinkage under dopaminergic therapy formed the indication subgroup 3 with 52 $(27.4 \%)$ patients and was distributed among the time periods A to $C$ in $29.0,26.6$, and $26.5 \%$ respectively (Fig. 1 and Table 3).

Patient's individual decision for a primary surgical treatment established the indication in $23(12.1 \%)$ cases (subgroup 4) and was found distributed among the time periods $\mathrm{A}$ to $\mathrm{C}$ in $6.5,11.4$, and $20.4 \%$ respectively (Fig. 1 and Table 3 ).

Fifty $(26.3 \%)$ patients had prolactinomas with dominant cystic tumor parts and formed indication subgroup 5 with a distribution among the time periods A to $\mathrm{C}$ in 19.4 , 29.1, and 30.6\% respectively (Fig. 1 and Table 3 ).

In combination, the 'classical' subgroups 1-3 set the operative indication in our patients with prolactinomas over the last 15 years in a decreasing number of $74.2 \%$ (time period A), 59.5\% (time period B), and $49.0 \%$ (time period $\mathrm{C})$. By contrast, the number of patients representing the defined 'modern' indication subgroups increased progressively throughout the last 15 years with $25.8,40.5$, and $51.0 \%$ during time periods A to C respectively (Fig. 2).

The mean age of patients showed no difference between the 'classical' and the 'modern' subgroup (35 years) and was equal to the mean age of patients concerning the whole series.

\section{Discussion}

There is no doubt that medical treatment of PRL-secreting pituitary adenomas is the mainstay of primary therapy. The introduction of modern DA during the last three decades has strengthened this position. Nevertheless,

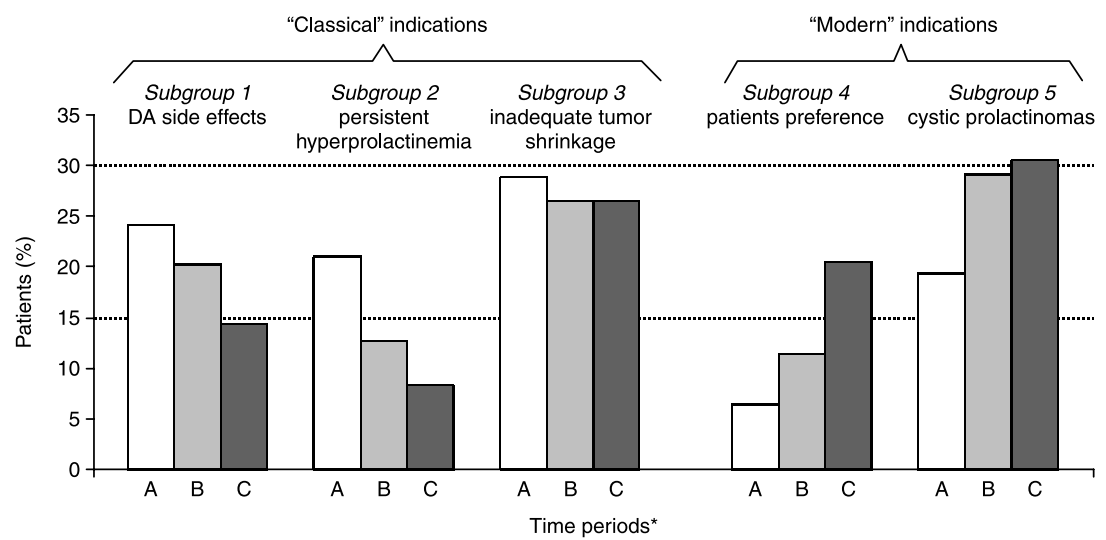

Figure 1 Operative therapy of prolactinomas at the Department of Neurosurgery, University of Erlangen-Nuremberg between 1990 and 2005 - Surgical indications sorted by time periods. *(A) 1990-1995; (B) 1995-2000; (C) 2000-2005. 
Table 3 Surgical indication subgroups and their distribution among time periods A-C $(n=190)$.

\begin{tabular}{|c|c|c|c|c|c|c|}
\hline & \multicolumn{6}{|c|}{ Surgical indication subgroups ${ }^{a}$} \\
\hline & $\begin{array}{c}1 \\
n=38\end{array}$ & $\begin{array}{c}2 \\
n=27\end{array}$ & $\begin{array}{c}3 \\
n=52\end{array}$ & $\begin{array}{c}4 \\
n=23\end{array}$ & $\begin{array}{c}5 \\
n=50\end{array}$ & $\underset{n=190}{\Sigma}$ \\
\hline Time period A (1990-mid-1995) & $15(24.2 \%)$ & $13(21 \%)$ & $18(29.0 \%)$ & $4(6.5 \%)$ & $12(19.4 \%)$ & 62 \\
\hline Time period B (mid-1995-mid-2000) & $16(20.3 \%)$ & $10(12.7 \%)$ & $21(26.6 \%)$ & $9(11.4 \%)$ & $23(29.1 \%)$ & 79 \\
\hline Time period C (mid-2000-2005) & $7(14.3 \%)$ & $4(8.2 \%)$ & $13(26.5 \%)$ & $10(20.4 \%)$ & $15(30.6 \%)$ & 49 \\
\hline
\end{tabular}

${ }^{a} 1$, side effects of DA therapy; 2 , failure of prolactin level normalization; 3 , failure of sufficient adenoma shrinkage; 4 , patient's preference for surgical treatment; 5 , cystic prolactinomas.

starting in the 1970s pituitary surgery has also developed tremendously from a technical standpoint and specialized centers have emerged throughout the years. However, transsphenoidal surgery is currently only seen as a secondline treatment in prolactinomas with very distinct indications accepted so far. It was only recently at the 9th International Pituitary Congress in San Diego in 2005 that an interdisciplinary committee of experts, also including one of the senior authors of this manuscript, has formulated management guidelines for prolactinomas (35). This committee finalized that non-responders to dopaminergic therapy, patients with intolerable adverse effects of DA therapy, patients with CSF fistulas under DA, or patients with rapidly progressive neurological deficits are candidates for a surgical intervention. These patients also represent the 'classical' indication subgroups in this paper. As a result of the above consensus statement, we felt the necessity for a retrospective analysis of our surgical results and experience in the management of prolactinomas focusing on a possible shift of operative indications at a specialized centre during the last 15 years.

The overall normalization rate of morning fasting PRL levels concerning the whole series, thus including already initially unresectable invasive parasellar tumors and giant prolactinomas was $53.2 \%$ initially and $42.7 \%$ at the latest follow-up. By contrast, our analysis demonstrates that the remission rate of intrasellar (is) macroprolactinomas was favorable with $82.4 \%$ initially, and $72.5 \%$ at the latest follow-up. These remission rates are well comparable with

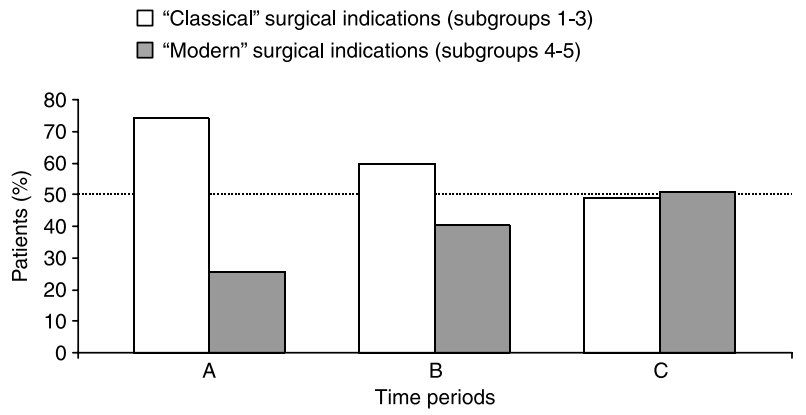

Figure 2 Operative treatment of prolactinomas at the Department of Neurosurgery, University of Erlangen-Nuremberg - Relative development of 'classical' and 'modern' surgical indications over the last 15 years. (A) 1990-1995; (B) 1995-2000; (C) 2000-2005. most of the results published for primary dopaminergic therapy (8).

Our data also demonstrate that transsphenoidal surgery for microprolactinomas in the hands of experienced pituitary surgeons is associated with an excellent remission rate of $91.3 \%$ (84.8\% follow-up remission) and a superior remission rate of $92.3 \%(87.2 \%$ follow-up remission) for the subgroup of purely intrasellar microadenomas. These results of surgical therapy are well comparable with the best rates of PRL normalization published for medical therapy with cabergoline or quinagolide $(8,15-20)$. Concerning microprolactinomas, the overall recurrence rate was $7.1 \%$ and therefore set in the lower range of recurrence rates published before $(27,33,34,37,38)$. The most favorable recurrence rate was detected for the subgroup of purely intrasellar located microprolactinomas with $5.6 \%$. The overall recurrence rate of $18.7 \%$ compares favorably with the recurrence rates after withdrawal of Cabergoline therapy in microprolactinomas and macroprolactinomas reported in a large prospective study by Colao et al. (21) or the retrospective data of Biswas et al. (22). Moreover, the recurrence rate in the subgroup of patients with remnant tumor on MRI before withdrawal was reported with 42 and $78 \%$ in micro- and macroadenomas respectively after all. Although the morbidity of our surgical series was low with $3.8 \%$ (1.8\% in microprolactinomas), we have to report new anterior pituitary deficiencies in $7.0 \%$ of patients, exclusively in macroprolactinomas. By contrast, excluding hyperprolactinemia, decompression of the pituitary gland by selective transsphenoidal adenomectomy restored pituitary functions in $14.6 \%$.

With regard to the main intention of our study, namely a potential refinement of the current interdisciplinary guidelines from a neurosurgical standpoint, our series additionally indicate the need to pay extra attention to the subgroup of cystic intrasellar and suprasellar (s1) tumors with a PRL normalization rate of $80.0 \%$ comparable with the remission rates in microprolactinomas and the most favorable results published for primary medical therapy.

The combined analysis of the three 'classical' indication subgroups in contrast to the two 'modern' subgroups revealed a trend in opposite directions over the last 15 years (Fig. 2). In conclusion, the continuously decreasing 
number of patients admitted to our neuroendocrineneurosurgical outpatient clinic who eventually needed transsphenoidal surgery because of a persistent substantial hyperprolactinemia despite DA therapy or because of intolerable adverse effects displays the positive impact of modern DA like quinagolide and mainly cabergoline (Fig. 1). The number of non-responders regarding tumor size remained virtually unchanged.

On the other hand, we have seen a continuously increasing number of younger patients who wish for a potential definite surgical solution when they are informed that they may maintain medical therapy for a longer time (Fig. 1). Regarding this decision, we also have to retain in memory the Kaplan-Meier estimate of the 5-year recurrence rates after cabergoline withdrawal in micro- and macroprolactinomas with adenoma remnants on MRI (42 and $78 \%$ respectively) despite the very convincing rates of persistent normoprolactinemia after withdrawal of cabergoline (microprolactinomas 70\% and macroprolactinomas 64\%) reported by same group (21). It seemed that the individual preference for a surgical therapy was especially prevalent in young female patients who have to change back or were already initially started with bromocriptine because of the lack of adequate safety data during pregnancy concerning the modern DA. With regard to primary surgical therapy in prolactinomas, it will also be interesting to follow the potential impact of the significantly increased frequency of mitral regurgitation reported in patients using cabergoline in high doses for Parkinson's disease (39), once these data have been looked-over for lower cabergoline dosages frequently used in the therapy of prolactinomas.

In conclusion, our series descriptively displays the current strengths and weaknesses of surgical therapy of prolactinomas at a specialized center. Thereby, our remission data not only confirm the already interdisciplinarily accepted surgical indications mentioned above, but also, in our opinion, at least justify the discussion about additional (maybe best called 'modern') indications for a primary surgical therapy in the management of prolactinomas. The excellent follow-up remission rates in microprolactinomas, intrasellar macroprolactinomas, and cystic is and s1prolactinomas in the hands of experienced pituitary surgeons emphasize the value of primary transsphenoidal surgery as a discussion worthy alternative to dopamine agonist therapy in young patients harboring the above prolactinoma subtypes. Of course, we want to emphasize that these data cannot be generally extrapolated to all neurosurgical centers. At the moment, the suggested alternative management strategies should be the result of an interdisciplinary discussion of each individual case between experienced endocrinologists and pituitary surgeons. However, we think that maybe the time has come for a randomized (preferable multicentre) trial to finally prove the alternative value of primary surgical treatment of prolactinomas in patients with microprolactinomas and/or cystic tumors.

\section{References}

1 Ciccarelli A, Daly AF \& Beckers A. The epidemiology of prolactinomas. Pituitary 20058 3-6.

2 Vance ML \& Thorner MO Prolactin: hyperprolactinemic syndromes and management. In Ed. LJ De Groot, Textbook of Endocrinology, edn 2, WB Saunders Company, Philadelphia, London, Toronto, Mexico City, Rio de Janeiro, Sydney, Tokyo, Hong Kong, 1989, pp. 408-418.

3 Thorner MO, McNeilly AS, Hagan C \& Besser GM. Long-term treatment of galactorrhoea and hypogonadism with bromocriptine. BMJ 19742 419-422.

4 Werder K, Fahlbusch R, Landgraf R, Pickardt CR, Rjosk HK \& Scriba PC. Treatment of patients with prolactinomas. Journal of Endocrinological Investigation 1978 1 47-58.

5 Bevan JS, Webster J, Burke CW \& Scanlon MF. Dopamine agonists and pituitary tumor shrinkage. Endocrine Reviews 199213 220-240.

6 Molitch ME, Thorner MO \& Wilson C. Management of prolactinomas. Journal of Clinical Endocrinology and Metabolism 199782 996-1000.

7 Webster J. A comparative review of the tolerability profiles of dopamine agonists in the treatment of hyperprolactinaemia and inhibition of lactation. Drug Safety 199614 228-238.

8 Webster J, Piscitelli G, Polli A, Ferrari Cl, Ismail I \& Scanlon MF. For the Cabergoline Comparative Study Group. New England Journal of Medicine, 1994 904-909.

9 Boyd A. Bromocriptine and psychosis: a literature review. Psychiatric Quarterly 199566 87-95.

10 Colao A, Vitale G, Cappabianca P, Briganti F, Ciccarelli A, De Rosa M, Zarrilli S \& Lombardi G. Outcome of cabergoline treatment in men with prolactinoma: effects of a 24-month treatment on prolactin levels, tumor mass, recovery of pituitary function, and semen analysis. Journal of Clinical Endocrinology and Metabolism 200489 1704-1711.

11 Molitch ME. Medical management of prolactin-secreting pituitary adenomas. Pituitary 20025 55-65.

12 Webster J, Piscitelli G, Polli A, D’Alberton A, Falsetti L, Ferrari C, Fioretti P, Giordano G, L'Hermite M, Ciccarelli E, Crogsignani PG, Dedecco L, Fandini R, Faglia G, Flamigni C, Tamburrano G, Ismail I \& Scanlon MF. The efficacy and tolerability of long-term cabergoline therapy in hyperprolactinaemic disorders: an open, uncontrolled, multicentre study. European Multicentre Cabergoline Study Group. Clinical Endocrinology 199339 323-329.

13 Colao A, Merola B, Sarnacchiaro F, Di Sarno A, Landi ML, Marzullo P, Cerbone G, Ferone D \& Lombardi G. Comparison among different dopamine-agonists of new formulation in the clinical management of macroprolactinomas. Hormone Research $19955222-228$.

14 Colao A, Vitale G, Cappabianca P, Briganti F, Ciccarelli A, De Rosa M, Zarrilli S \& Lombardi G. Outcome of cabergoline treatment in men with prolactinoma: effects of a 24-month treatment on prolactin levels, tumor mass, recovery of pituitary function, and semen analysis. Journal of Clinical Endocrinology and Metabolism 20044 1704-1711.

15 Verhelst J, Abs R, Maiter D, van den Bruel A, Vandeweghe M, Velkeniers B, Mockel J, Lamberigts G, Petrossians P, Coremans P, Mahler C, Stevenaert A, Verlooy J, Raftopoulos C \& Beckers A. Cabergoline in the treatment of hyperprolactinemia: a study in 455 patients. Journal of Clinical Endocrinology and Metabolism, $19992518-2522$.

16 Colao A, De Rosa M, Sarnacchiaro F, Di Sarno A, Landi ML, Iervolino E, Zarrilli S, Merola B \& Lombardi G. Chronic treatment with CV 205-502 restores the gonadal function in hyperprolactinemic males. European Journal Endocrinology 1996135 548-552. 
17 Colao A, di Sarno A, Pivonello R, di Somma C \& Lombardi G. Dopamine receptor agonists for treating prolactinomas. Expert Opinion on Investigational Drugs 200211 787-800.

18 Colao A, Merola B, Sarnacchiaro F, Di Sarno A, Landi ML, Marzullo P, Cerbone G, Ferone D \& Lombardi G. Comparison among different dopamine-agonists of new formulation in the clinical management of macroprolactinomas. Hormone Research $199544222-228$.

19 Serri O, Beauregard H, Lesage J, Pedneault L, Comtois R, Jilwan N, Somma M, Vachon L \& Brownell J. Long term treatment with CV 205-502 in patients with prolactin-secreting pituitary macroadenomas. Journal of Clinical Endocrinology and Metabolism 1990 71 682-687.

20 van der Lely AJ, Brownell J \& Lamberts SW. The efficacy and tolerability of CV 205-502 (a nonergot dopaminergic drug) in macroprolactinoma patients and in prolactinoma patients intolerant to bromocriptine. Journal of Clinical Endocrinology and Metabolism 199172 1136-1141.

21 Colao A, Di Sarno A, Cappabianca P, Di Somma C, Pivonello R \& Lombardi G. Withdrawal of long-term cabergoline therapy for tumoural and nontumoural hyperprolactinemia. New England Journal of Medicine $20033492023-2033$.

22 Biswas M, Smith J, Jadon D, McEwan P, Rees DA, Evans LM, Scanlon MF \& Davies JS. Long-term remission following withdrawal of dopamine agonist therapy in subjects with microprolactinomas. Clinical Endocrinology 200563 26-31.

23 Nomikos P, Buchfelder M \& Fahlbusch R. Current management of prolactinomas. Journal of Neuro-Oncology 200154 139-150.

24 Abe T \& Ludecke DK. Transnasal surgery for prolactin-secreting pituitary adenomas in childhood and adolescence. Surgical Neurology 200257 369-378 (discussion 378-379).

25 Couldwell WT, Rovit RL \& Weiss MH. Role of surgery in the treatment of microprolactinomas. Neurosurgery Clinics of North America 200314 89-92 (vii).

26 Feigenbaum SL, Downey DE, Wilson CB \& Jaffe RB. Transsphenoidal pituitary resection for preoperative diagnosis of prolactin-secreting pituitary adenoma in women: long term follow-up. Journal of Clinical Endocrinology and Metabolism 1996 81 1711-1719.

27 Massoud F, Serri O, Hardy J, Somma M \& Beauregard H. Transsphenoidal adenomectomy for microprolactinomas: 10 to 20 years of follow-up. Surgical Neurology $199645341-346$.

28 Mortini P, Losa M, Barzaghi R, Boari N \& Giovanelli M. Results of transsphenoidal surgery in a large series of patients with pituitary adenoma. Neurosurgery 200556 1222-1233 (discussion 1233).
29 Post KD, Biller BJ, Adelman LS, Molitch ME, Wolpert SM \& Reichlin S. Selective transsphenoidal adenomectomy in women with galactorrhea-amenorrhea. Journal of the American Medical Association 1979242 158-162.

30 Randall RV, Laws ER, Jr, Abboud CF, Ebersold MJ, Kao PC \& Scheithauer BW. Transsphenoidal microsurgical treatment of prolactin-producing pituitary adenomas. Results in 100 patients. Mayo Clinic Proceedings 198358 108-121.

31 Turner HE, Adams CB \& Wass JA. Trans-sphenoidal surgery for microprolactinoma: an acceptable alternative to dopamine agonists? European Journal of Endocrinology 1999140 43-47.

32 Tyrrell JB, Lamborn KR, Hannegan LT, Applebury CB \& Wilson CB. Transsphenoidal microsurgical therapy of prolactinomas: initial outcomes and long-term results. Neurosurgery 1999 44 254-261 (discussion 261-263).

33 Wolfsberger S, Czech T, Vierhapper H, Benavente R \& Knosp E. Microprolactinomas in males treated by transsphenoidal surgery. Acta Neurochirurgica 2003145 935-940 (discussion 940-941).

34 Losa M, Mortini P, Barzaghi R, Gioia L \& Giovanelli M. Surgical treatment of prolactin-secreting pituitary adenomas: early results and long-term outcome. Journal of Clinical Endocrinology and Metabolism 200287 3180-3186.

35 Casanueva FF, Molitch ME, Schlechte JA, Abs R, Bonert V, Bronstein MD, Brue T, Cappabianca P, Colao A, Fahlbusch R, Fideleff H, Hadani M, Kelly P, Kleinberg D, Laws ER, Marek J, Scanlon MF, Sobrinho LG, Wass JA \& Giustina A. Guidlines of the Pituitary Society for the diagnosis and management of prolactinomas. Clinical Endocrinology 200665 265-273.

36 DeLellis RA, Lloyd RV, Heitz PU, Eng C. (Eds) Pathology and Genetics of Tumours of Endocrine Organs. Oxford University Press, 2004.

37 Thomson JA, Davies DL, McLaren E \& Teasdale GM. Ten year follow-up of microprolactinoma treated by transsphenoidal surgery. BMJ 1994309 1409-1410.

38 Serri O, Rasio E, Beauregard H, Hardy J \& Somma M. Recurrence of hyperprolactinemia after selective adenomectomy in women with prolactinoma. New England Journal of Medicine 1983309 280-283.

39 Zanettini R, Antonini A, Gatto G, Gentile R, Tesei S \& Pezzoli G. Valvular heart disease and the use of dopamine agonists for Parkinson's disease. New England Journal of Medicine $2007 \mathbf{3 5 6}$ $39-46$.

Received 5 October 2007

Accepted 14 October 2007 